\title{
Targeted Gene Integration into Nuclear Genome of Microalgae Using Cre/loxP Recombination System
}

\author{
Kazuki SHIRAKAWA ${ }^{1}$, Yoshinori KAWABE ${ }^{1}$, Guan $\mathrm{HUANG}^{2}$, Akira ITO ${ }^{1}$, and Masamichi KAMIHIRA K, $^{\text {2* }}$ \\ ${ }^{1}$ Faculty of Engineering, Kyushu University, 744 Motooka, Nishi-ku, Fukuoka 819-0395, Japan \\ ${ }^{2}$ Graduate School of Systems Life Sciences, Kyushu University, 744 Motooka, Nishi-ku, Fukuoka 819-0395, Japan
}

\begin{abstract}
Genetically modified microalgae have been expected to be a useful tool for bioenergy and recombinant protein production. However, random integration of transgene in the microalgae nuclear genome is susceptible to gene silencing of heterologous gene expression. Here, we attempted to perform targeted gene integration into a pre-determined nuclear genomic site of Chlamydomonas reinhardtii using Cre/loxP recombination system for stable transgene expression. We constructed an expression vector plasmid encoding reporter genes (zeocin resistant gene and green fluorescent protein gene; Zeo-2A-GFP) and mutated loxP to generate founder cells. A donor vector encoding IFNa-4 and paromomycin resistant genes flanked by corresponding mutated loxPs was constructed and introduced into founder cells together with a Cre expression vector. The optimal ratio of donor vector to Cre expression vector was determined by counting the number of paromomycin resistant colonies. For the established clones, the targeted integration was confirmed by genomic PCR using various specific primer sets. Target genes in the donor vector could be integrated into the expected genomic site of $C$. reinhardtii using Cre/loxP system. RT-PCR revealed that IFNa-4 was expressed in five independent transgenic cell lines tested. This result suggests that Cre-based cell engineering is a promising approach to generate smart microalgae expressing foreign genes.
\end{abstract}

\section{Introduction}

Photosynthetic microalgae have attracted great interest as a sustainable green cell factory and have the potential to revolutionize many biotechnology fields including nutrition, pharmaceuticals and biofuels (Rasala and Mayfield, 2015). Genetically engineered microalgae can be expected to be more useful in bioenergy and recombinant protein production. To generate genetically engineered microalgae, a foreign DNA fragment should be incorporated into the cell nuclear or chloroplast genome through successful transformation events. Protein expression from the nuclear genome has the advantage that extracellular secretion and appropriate post-translational modification can be performed, although the expression level from the nuclear genome tends to be low compared to the chloroplast expression level. However, random integration of transgene into the nuclear genome of microalgae causes gene silencing of heterologous transgene expression depending on the insertion position. Thus, construction of a stable gene expression system is desired (Scranton et al., 2015).

Several attempts have been made to artificially generate mutants that are deficient in silencing mechanisms and that can efficiently accumulate proteins. Neupert et al. (2009) generated Chlamydomonas reinhardtii mutants expressing transgenes at high levels.
Nevertheless, when recombinant proteins are produced from the nuclear genome, the major issue of transgene silencing remains unimproved (Specht et al., 2010).

Among the site-specific gene recombination systems, the Cre/loxP system has been well studied. The Cre recombinase derived from bacteriophage P1 recognizes the $34 \mathrm{bp}$ target sequence lox $P$, and catalyses a recombination reaction between the two loxP sites. This system has been used in the field of gene function analysis such as the generation of conditional knockout mice for deletion of target genes under specific conditions (Haenebalcke et al., 2013). Many mutated $\operatorname{lox} P$ sites have been reported to alter reaction kinetics. Mutant $\operatorname{lox} P$ sites with arm mutations representative of lox71 and lox66 make it possible to facilitate the integration reaction. Substitutional mutations within the spacer region of loxP can be used to provide the specificity of recombination, leading to recombinasemediated cassette exchange (RMCE).

We have previously developed an accumulative gene integration system (AGIS) using Cre and mutant loxP sites, in which repeated integration of transgenes into a predetermined genomic site is possible (Kameyama et al., 2010; Obayashi et al., 2012). This system was applied for constructing producer cell lines for pharmaceutical protein production using Chinese hamster ovary (CHO) cells (Wang et al., 2017). Recently,

Corresponding author: kamihira@chem-eng.kyushu-u.ac.jp 
Cre-lox $P$-mediated site-specific gene recombination was used to delete transgene flanked by $\operatorname{lox} P$ sites in $C$. reinhardtii (Kasai and Harayama, 2016). However, there are no reports to apply for transgene integration using the Cre-loxP system in microalgae containing $C$. reinhardtii. In this study, we generated transgenic $C$. reinhardtii harboring a mutant lox $P$ site in the genome for transgene integration. Using the cells, the sitespecific integration efficiency using Cre recombinase and the expression of target gens were evaluated.

\section{Materials and Method}

\subsection{Cells and culture medium}

C. reinhardtii strain $\mathrm{CC}-406$ (wild type, $\mathrm{mt}^{-}$) was provided by the Chlamydomonas Resource Center (MN, USA). Cells were mixotrophically cultivated in T-25 flask (Greiner Bio-One, Waltham, MA, USA), at $25^{\circ} \mathrm{C}$ in Tris-acetate phosphate (TAP) medium under moderate and constant white fluorescent light $(100 \mu \mathrm{mol}$ photons $\mathrm{m}^{-2} \mathrm{~s}^{-1}$, Model CR-300L, Tomy, Tokyo, Japan) with gentle shaking (50 rpm, Wave-SI, Model 0054334-000, Taitec, Saitama, Japan).

\subsection{Plasmid construction}

DNA sequences of mutant loxP sites used in this study are summarized in Table 1. pChlamy3 (Invitrogen, Carlsbad, CA, USA) was digested with XhoI and BamHI, blunted with Klenow enzyme (Fujifilm Wako Pure Chemical Corporation, Osaka, Japan) and then selfligated to generate pChlamy to remove the hygromycin resistant gene expression unit. A DNA fragment encoding zeocin resistant gene $\left(z e o^{r}\right)$, foot-and-mouth disease virus-derived $2 \mathrm{~A}$ self-cleaving peptide (2A) and GFP genes were chemically synthesized with optimized codon usage for $C$. reinhardtii. Mutant loxP (loxP1) was placed upstream $z e o^{r}$. The gene fragments were ligated into pChlamy to generate pChlamy/Z2G.

To construct a donor vector encoding target genes for integration using the Cre-loxP system, the DNA fragment encoding an IFN $\alpha-4$ expression unit and paromomycin resistance gene $\left(\right.$ paro $\left.^{r}\right)$ flanked by compatible mutant loxPs (loxP2-loxP4) were inserted into pMC-R2 (Wang et al., 2018) to generate pDonor/Paro-IFN.

A Cre recombinase gene chemically synthesized according to the $C$. reinhardtii usage and constitutive Hsp70A-RbcS2 (AR) promoter fragment derived from pVenus (kindly gifted from Prof. Betenbaugh of Johns Hopkins University, USA), were inserted into pBluescript (Stratagene, La Jolla, CA, USA) to generate pAR-Cre.

The sequences of all DNA constructs derived from chemically synthesized oligonucleotides and PCR products were confirmed using a DNA sequencer (Prism 3130 Genetic Analyzer, Applied Biosystems, Foster City, CA, USA).

\subsection{Generation of founder cells and Cre- mediated transgenic cell lines}

Plasmid vectors were introduced into cells using an electroporation device (NEPA21, Nepagene, Chiba, Japan). Briefly, cells were grown to $5.0 \times 10^{6}$ cells $/ \mathrm{mL}$ in TAP medium. The cells were counted using cell counting device with Cy5 flurescence (Countess, Invitrogen) or Evans blue staining method. Subsequently, $3.8 \times 10^{6}$ cells were harvested by centrifugation and suspended in $38 \mu \mathrm{L}$ of TAP medium supplemented with $40 \mathrm{mM}$ sucrose (TAP/sucrose). To generate founder cells, electroporation was performed under the voltage conditions shown in Table 2, using $200 \mathrm{ng}$ of linearized pChlamy/Z2G by digesting with NotI-KpnI. After electroporation, the cells were cultured in $10 \mathrm{~mL}$ of $\mathrm{TAP} /$ sucrose for $24 \mathrm{~h}$ under dim light condition. The transgenic strains were selected directly on TAP/agar plates containing $15 \mathrm{mg} / \mathrm{L}$ zeocin (Invitrogen) and the plates were incubated under continuous fluorescent light (LED lamp $100 \mathrm{~V}, 9.5 \mathrm{~W}$, Tomy) at $25^{\circ} \mathrm{C}$ for $10-14$ days.

For Cre-mediated integration reaction, Chlamy/Z2G founder cells (\#1-3) prepared at a density of $3.8 \times 10^{6}$ cells were co-transfected with pDonor/ParoIFN (200 ng) and a Cre expression vector (pAR-Cre) (0$200 \mathrm{ng}$ ) by electroporation as described above except for screening on TAP/agar plates containing $10 \mathrm{mg} / \mathrm{L}$ paromomycin (Wako). After 10-14 d culture, paromomycin-resistant colonies were selected for establishing cell clones (Chlamy/C1-Chlamy/C5). Number of paromomycin-resistant colonies was counted for $1 \mathrm{~cm}^{2}$ of five areas and expressed as mean values with standard deviation of the mean. All clones obtained were analyzed by genomic PCR to confirm site-specific integration, as described below.

\subsection{Genomic PCR analysis}

Genomic DNA was extracted from cells using a genomic DNA preparation kit (MagExtractor ${ }^{\circledR}$, Toyobo, Osaka, Japan). The sequences to detect site-specific recombination were amplified by PCR from genomic DNA (50 ng) as the template using various specific primer sets. The PCR was initiated with DNA polymerase $\left(\right.$ Gflex $^{\circledR}$ DNA polymerase, Takara Bio, Kusatsu, Japan or KOD FX Neo, Toyobo).

\subsection{Isolation of total mRNA and RT-PCR analysis}

Total mRNA was isolated using an RNAiso plus (Takara Bio), according to the manufacturer's protocol. The RNA was reverse-transcribed into cDNA from $1 \mu \mathrm{g}$ total RNA using a ReverTra Ace First Strand cDNA synthesis kit (Toyobo) with Oligo(dT)20 primers. RT-PCR was performed using the following primers: 5'-CGA CAC CCA CTC TTT ACG CA-3' and 5'-AAG TGC TGT CCT CGT GCA TT-3' for $I F N \alpha-4$ and 5'- CGT CTG TGG GAC CTG AAC AC -3' and 5'-GCT CGC CAA TGG TGT ACT TG -3 ' for housekeeping gene CLBP to evaluate the transgene expression. To evaluate the 
expression level of $I F N \alpha-4$, the band densities in electrophoresis images were quantified using ImageJ software V.1.52a (National Institutes of Health, Bethesda, MD, USA).

Table 1. Sequences of wild-type and mutant loxP sites

\begin{tabular}{cccc}
\hline \multirow{2}{*}{ Symbol } & \multicolumn{3}{c}{ Sequence $\left(5^{\prime} \rightarrow 3^{\prime}\right)$} \\
\cline { 2 - 4 } & $\begin{array}{c}\text { Left-arm } \\
\text { region }\end{array}$ & $\begin{array}{c}\text { Spacer } \\
\text { region }\end{array}$ & $\begin{array}{c}\text { Right-arm } \\
\text { region }\end{array}$ \\
\hline loxP & ATAACTTCGTATA & GCATACAT & TATACGAAGTTAT \\
loxP1 & ATAACTTCGTATA & AAGTATCC & TATACGAACGGTA \\
loxP2 & TACCGTTCGTATA & $\underline{\text { AAGTATCC }}$ & TATACGAAGTTAT \\
loxP3 & TACCGTTCGTATA & $\underline{\text { AAGTATCC }}$ & TATACGAACGGTA \\
loxP4 & ATAACTTCGTATA & ACCATAAT & TATACGAA $\overline{\text { ACGGTA }}$ \\
loxP5 & TACCGTTCGTATA & A ACCATAAT & TATACGAAGTTAT \\
\hline
\end{tabular}

Mutated sequences are underlined.

Table 2. Conditions of electroporation [NEPA21]

\begin{tabular}{ccccccc}
\hline $\begin{array}{c}\text { Pulse } \\
\text { phase }\end{array}$ & $\begin{array}{c}\text { Voltage } \\
{[\mathrm{V}]}\end{array}$ & $\begin{array}{c}\text { Pulse } \\
\text { length } \\
{[\mathrm{msec}]}\end{array}$ & $\begin{array}{c}\text { Pulse } \\
\text { Interval } \\
{[\mathrm{msec}]}\end{array}$ & $\begin{array}{c}\text { Number } \\
\text { of } \\
\text { pulse }\end{array}$ & $\begin{array}{c}\text { Decay } \\
\text { rate } \\
{[\%]}\end{array}$ & Polarity \\
\hline Poring & 250 & 8 & 50 & 2 & 40 & + \\
Transfer & 20 & 50 & 50 & 5 & 40 & $+/-$ \\
\hline
\end{tabular}

\section{Results}

\subsection{Cell growth}

First, we confirmed whether it was possible to perform cell counting of $C$. reinhardtii in cultivation using an image-based cell counter with a fluorescence filter for chlorophyll fluorescence, and compared it with a conventional method using a hemocytometer. CC-406 WT strain was seeded at initial cell number $1.0 \times 10^{6}$ cells $/ \mathrm{mL}$ and cell number was measured for $7 \mathrm{~d}$. Figure 1 shows the growth curve between image-based cell counter (Countess) and hemocytometer. There was no significant difference in specific growth rate between the two methods (Countess, $0.76 \mathrm{~d}^{-1}$; hemocytometer, $0.84 \mathrm{~d}^{-}$ $\left.{ }^{1}\right)$ in the exponential growth phase (day1-3). This result indicates that an automatic cell counter is applicable to the measurement of $C$. reinhardtii cell number.

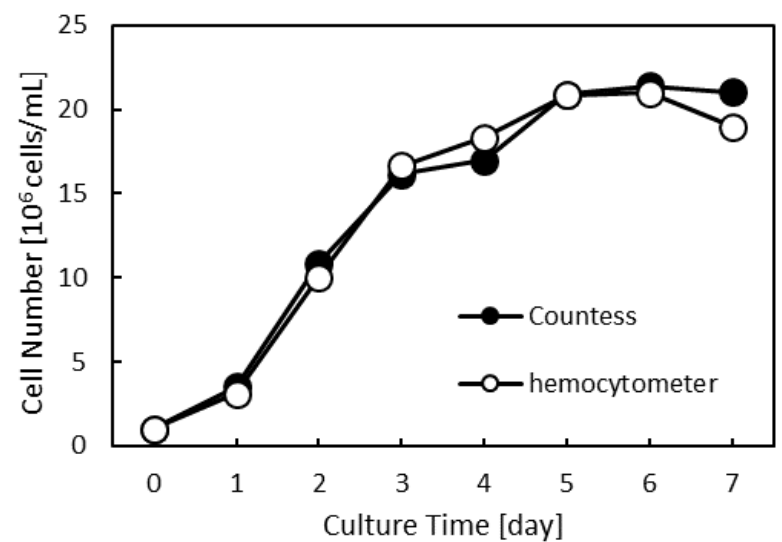

Figure 1. Growth curves of CC-406 WT C. reinhardtii strains. Black and white dots indicate the cell number measured by Countess and hemocytometer, respectively. Initial cell density for seeding was $1.0 \times$ $10^{6}$ cells $/ \mathrm{mL}$

\subsection{Establishment of founder cells}

A schematic drawing of the site-specific transgene integration into $C$. reinhardtii founder cell genome using Cre/loxP system is shown in Figure 2. After optimization of electroporation conditions for $C$. reinhardtii, pChlamy/Z2G plasmid including a mutant loxP site for target gene integration was introduced into $C$. reinhardtii cells using the electroporation method, and transgenic cells were screened using zeocin (Chlamy/Z2G). Genomic integration of pChlamy/Z2G sequences was confirmed by genomic PCR. To select stable cell clones established from the transgenic cell pool, GFP expression analysis was performed using FACS. FACS analysis revealed that Chlamy/Z2G\#1-3 clone stably expressed GFP under zeocin pressure. Thus, this clone was used as a founder cell for further gene integration experiment.

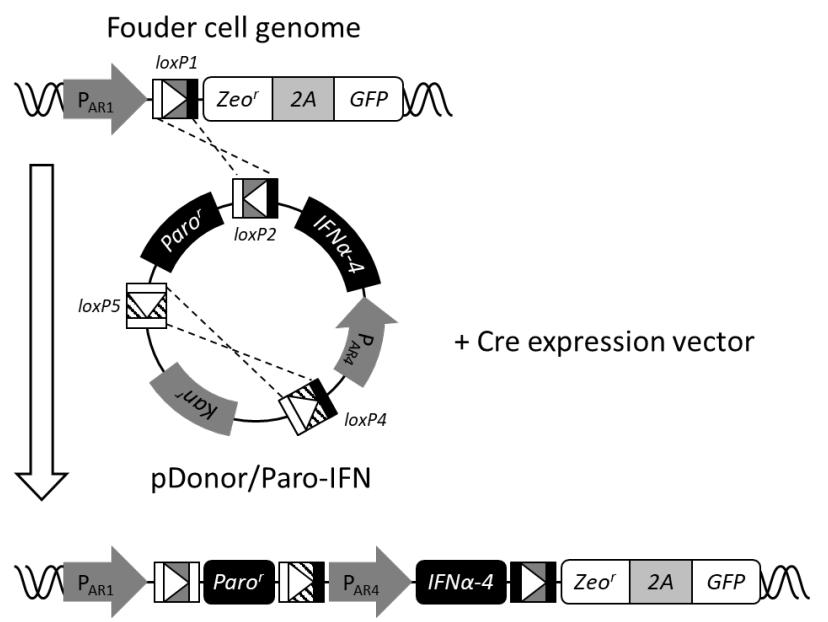

Figure 2. Schematic drawing of the site-specific transgene integration. Cre recombinase catalyzes a recombination reaction between two loxPs. During the reaction, transgene integration into the genome and deletion of the plasmid backbone are processed at the same time. Cre recombines between loxPs with the same spacer. The loxPs with doublemutated arms generated after recombination are no longer reactive for Cre-mediated recombination.

\subsection{Optimization of the ratio of donor vector and Cre expression vector}

In our previous report, integration efficiency of Cremediated reaction was dependent on the amount of Cre expression vector (Kameyama et al., 2010). Therefore, in order to determine the optimal ratio of donor vector and Cre expression vector amounts, cells were transfected with donor plasmid and various amount of Cre expression vector. Figure 3 shows the photographs of colonies formed after 10-14 d screening. By counting the number of paromomycin-resistant colonies, the optimal amount of Cre expression vector was $25 \mathrm{ng}$ (Table 3).

\subsection{Cre-mediated targeted integration of transgenes}


After Cre-mediated recombination, genomic DNA extracted from the established cell clones was subjected to PCR using various specific primer sets to confirm site-specific integration. The size of the amplified DNA fragments was consistent with the expected insertion size.

To check the expression of $I F N \alpha-4$, we extracted mRNA from the cells for RT-PCR analysis. Among fifty clones established, the IFN $\alpha-4$ expression was detected from five clones $(10 \%)$. The similar mRNA levels for $C L B P$ gene were detected for all samples, indicating that the similar levels of $I F N \alpha-4$ were expressed in the cells $\left(I F N \alpha-4 / C L B P=1.3-1.6\left[\right.\right.$ IFN $\alpha-4$ clones] vs. $3.2 \times 10^{-4}$ [founder]).

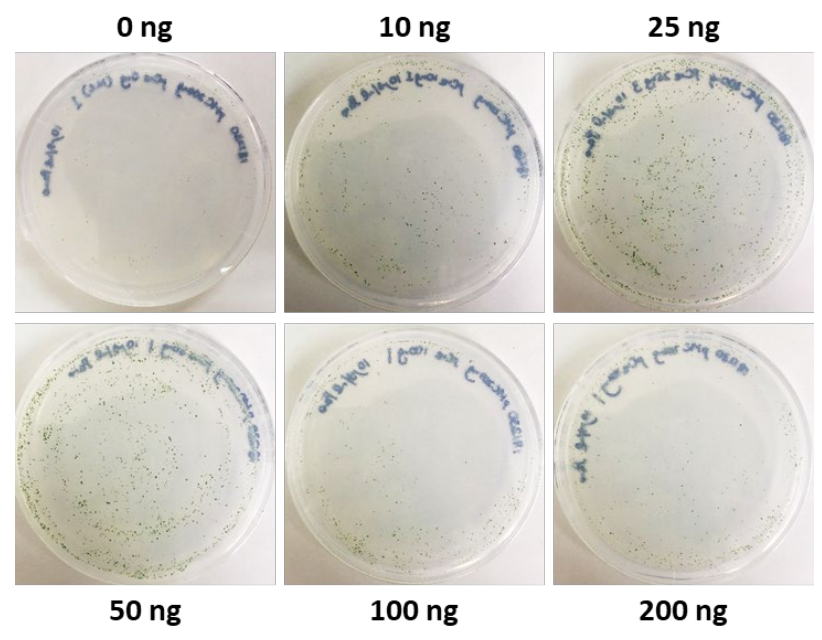

Figure 3. Comparison of targeted transgene integration efficiency depending on the amount of Cre expression vector

Table 3. The number of colonies per unit area

\begin{tabular}{cc}
\hline $\begin{array}{c}\text { Amount of Cre expression } \\
\text { vector }[\mathrm{ng}]\end{array}$ & The number of colonies $\left[/ \mathrm{cm}^{2}\right]$ \\
\hline 0 & $1.4 \pm 2.0$ \\
10 & $7.6 \pm 3.3$ \\
25 & $25.1 \pm 2.8$ \\
50 & $18.9 \pm 6.0$ \\
100 & $11.8 \pm 4.2$ \\
200 & $3.6 \pm 1.9$ \\
\hline
\end{tabular}

\section{Discussion}

The reference genome sequence including the nuclear genome, chloroplast and mitochondrial DNA of $C$. reinhardtii was elucidated in 2003 prior to other microalgae (Shrager et al., 2003). Nuclear transformation of microalgae is a first step in their use for biotechnological applications such as recombinant protein production or molecular modifications of specific cell metabolic pathways. In recent years, the use of genome editing tools such as CRISPR/Cas9 paves the way for user-friendly and efficient genome manipulation. There are some reports using the genome editing tools for $C$. reinhardtii (Shin et al., 2016). In contrast, although the Cre-loxP system is a conventional genetic manipulation procedure for site-specific recombination, the integration efficiency of transgenes is higher than that using the CRISPR/Cas9 (Shin et al., 2016; Wang et al., 2017; Kawabe et al., 2018). There have been reports using the Cre/loxP system for gene deletion in microalgae (Kasai and Harayama, 2016), but no report for gene integration. Here, we generated engineered $C$. reinhardtii cells with a mutant loxP introduced into the nuclear genome, as founder cells. Using the cells, Cremediated targeted integration of transgenes was possible.

The optimal ratio of donor vector and Cre expression vector was determined by counting the number of paromomycin resistant colonies. The integration efficiencies of transgenes into the genome of C. reinhardtii cells decreased as the amount of Cre expression vector increased ( $>25 \mathrm{ng}$ ). Excessive amount of Cre may have cytotoxic effects on cell growth (Baba et al., 2005). Alternatively, the recombination reaction of mutant loxPs with a double arm mutation may be promoted in the presence of excessive amounts of Cre, although the $\operatorname{lox} P$ sequences of both arm mutations should be non-reactive (Albert et al., 1995). A similar phenomenon has been observed for $\mathrm{CHO}$ cells in our previous report (Kameyama et al., 2010). However, low amount of Cre expression vector $(<25 \mathrm{ng})$ was insufficient for the integration reaction. Thus, the optimal ratio of donor plasmid and Cre expression vector could be determined as a $1: 8$.

Conventionally, a hemocytometer has been used to measure the number of microalgal cells. However, this technique takes time to obtain data and can results in user bias. In addition, for high cell density, measurement might not be easy. For counting microalgal cells using Parachlorella kessleri, it has been shown that the cell counter with fluorescence filter Countess II FL can correctly distinguish algae from other particles (Takahashi, 2018). This method can save measurement time as compared to conventional methods and provides accurate data. In fact, there was no significant difference in specific growth rates between using Countess and using a hemocytometer in $C$. reinhardtii, hence an automated cell counter is an efficient tool for measuring cell number of $C$. reinhardtii.

Many microalgae such as Dunaliella and chlorella are also known as generally recognized as safe (GRAS) because they do not produce harmful substances to humans. IFN $\alpha-4$, which contains 187 amino acids, is an oral composition for the prevention and treatment of periodontal disease (Ito et al., 2010). Transgenic strawberries (Interberry $\alpha^{\circledR}$ ) expressing IFN $\alpha-4$ have been developed for gingivitis reducing agent for dogs. We also focused on canine IFN $\alpha-4$ as a recombinant protein produced in transgenic microalgae. RT-PCR revealed that $I F N \alpha-4$ was expressed in five transgenic cell lines tested. The expression level of $I F N \alpha-4$ was similar in the five cell lines. However, the target protein was produced in culture supernatant at a low level of $\mathrm{pg} / \mathrm{mL}$ (data not shown). For obtaining higher expression of transgenes, establishment of screening procedure of stable founder cells, improvement of expression units including promoter and splicing (Baier et al., 2018a) and use of a suitable secretion signal sequence (Baier et al., $2018 b$ ) are necessary. For the establishment of producer cells, we have developed an accumulative gene 
integration system (AGIS) based on Cre/loxP gene recombination system. This integration system enables repeated integration of multiple transgenes into a predetermined site (Kameyama et al., 2010; Obayashi et al., 2012). A screening of "hotspot" genomic loci followed by retargeting and repeated integration of transgenes is possible using AGIS. The method can be used to establish stable and high productive microalgae.

In conclusion, targeted integration of transgenes into the nuclear genome of $C$. reinhardtii was possible using Cre/loxP system. This result suggests that Crebased cell engineering is a promising approach to generate smart microalgae expressing foreign genes.

\section{References}

Albert, H., E. C. Dale, E. Lee, and D. W. Ow; "Sitespecific Integration of DNA into Wild-type and Mutant lox Sites Placed in the Plant Genome," Plant J., 7, 649659 (1995)

Baba, Y., M. Nakano, Y. Yamada, I. Saito, and Y. Kanegae; "Practical Range of Effective Dose for Cre Recombinase-expressing Recombinant Adenovirus without Cell Toxicity in Mammalian Cells," Microbiol. Immunol., 49, 559-570 (2005)

Baier, T., J. Wichmann, O. Kruse, and K. J. Lauersen; "Intron-containing Algal Transgenes Mediate Efficient Recombinant Gene Expression in the Green Microalga Chlamydomonas reinhardtii," Nucleic Acids Res., 46, 6909-6919 (2018a)

Baier, T., D. Kros, R. C. Feiner, K. J. Lauersen, K. M. Müller, and O. Kruse; "Engineered Fusion Proteins for Efficient Protein Secretion and Purification of a Human Growth Factor from the Green Microalga Chlamydomonas reinhardtii," ACS Synth. Biol., 7, 25472557 (2018b)

Haenebalcke, L., S. Goossens, M. Naessens, N. Kruse, M. Farhang, S. Bartunkova, K. Haigh, T. Pieters, P. Dierickx, B. Drogat, O. Nyabi, D. Wirth, and J. Haigh; "Efficient ROSA26-based Conditional and/or Inducible Transgenesis Using RMCE-compatible F1 Hybrid Mouse Embryonic Stem Cells," Stem Cell Rev., 9, 774785 (2013)

Ito, A., E. Isogai, K. Yoshioka, K. Sato, N. Himeno, and T. Gotanda; "Ability of Orally Administered IFN- $\alpha 4$ to Inhibit Naturally Occurring Gingival Inflammation in Dogs," J. Vet. Med. Sci., 72, 1145-1151 (2010)

Kasai, Y. and S. Harayama; "Construction of MarkerFree Transgenic Strains of Chlamydomonas reinhardtii Using a Cre/loxP-Mediated Recombinase System," PLoS One, 11, e0161733 (2016)

Kameyama, Y., Y. Kawabe, A. Ito, and M. Kamihira; "An Accumulative Site-specific Gene Integration System Using Cre Recombinase-mediated Cassette Exchange," Biotechnol. Bioeng., 105, 1106-1114 (2010)
Kawabe, Y., S. Komatsu, M. Murakami, A. Ito, T. Sakuma, T. Nakamura, T. Yamamoto, and M. Kamihira; "Targeted Knock-in of an scFv-Fc Antibody Gene into the hprt Locus of Chinese Hamster Ovary Cells Using CRISPR/Cas9 and CRIS-PITCh Systems," J. Biosci. Bioeng., 125, 599-605 (2018)

Neupert, J., D. Karcher, and R. Bock; "Generation of Chlamydomonas Strains That Efficiently Express Nuclear Transgenes," Plant J., 57, 1140-1150 (2009)

Obayashi, H., Y. Kawabe, H. Makitsubo, R. Watanabe, Y. Kameyama, S. Huang, Y. Takenouchi, A. Ito, and M. Kamihira; "Accumulative Gene Integration into a PreDetermined Site Using Cre/loxP," J. Biosci. Bioeng., 113, 381-388 (2012)

Rasala, B. A. and S. P. Mayfield; "Photosynthetic Biomanufacturing in Green Algae; Production of Recombinant Proteins for Industrial, Nutritional, and Medical Uses," Photosynth. Res., 123, 227-239 (2015)

Scranton, M. A., J. T. Ostrand, F. J. Fields, S. P. Mayfield; "Chlamydomonas as a Model for Biofuels and Bio-products Production," Plant J., 82, 523-531 (2015)

Shin, S-E., J-M. Lim, H. K. Koh, E. K. Kim, N. K. Kang, S. Jeon, S. Kwon, W-S. Shin, B. Lee, K. Hwangbo, J. Kim, S. H. Ye, J-Y. Yun, H. Seo, H-M. Oh, K-J. Kim, JS. Kim, W-J. Jeong, Y. K. Chand, and B-R. Jeong; "CRISPR/Cas9-induced knockout and knock-in mutations in Chlamydomonas reinhardtii," Sci. Rep., 6, 27810 (2016)

Shrager, J., C. Hauser, C. W. Chang, E. H. Harris, J. Davies, J. McDermott, R. Tamse, Z. Zhang, and A. R. Grossman; "Chlamydomonas reinhardtii Genome Project. A Guide to the Generation and Use of the cDNA Information," Plant Physiol., 131, 401-408 (2003)

Specht, E., S. Miyake-Stoner, and S. P. Mayfield; "Micro-algae Come of Age as a Platform for Recombinant Protein Production," Biotechnol. Lett., 32, 1373-1383 (2010)

Takahashi, T.; “Applicability of Automated Cell Counter with a Chlorophyll Detector in Routine Management of Microalgae,"Sci. Rep., 8, 4967 (2018)

Wang, X., Y. Kawabe, R. Kato, T. Hada, A. Ito, Y. Yamana, M. Kondo, and M. Kamihira; "Accumulative scFv-Fc Antibody Gene Integration into the hprt Chromosomal Locus of Chinese Hamster Ovary Cells," J. Biosci. Bioeng., 124, 583-590 (2017)

Wang, X., Y. Kawabe, T. Hada, A. Ito, and M. Kamihira; "Cre-Mediated Transgene Integration in Chinese Hamster Ovary Cells Using Minicircle DNA Vectors," Biotechnol. J., 13, e1800063 (2018) 